\title{
PREDIÇÃO DE SÉRIES TEMPORAIS DE PARÂMETROS DE REDE WCDMA - HSPA
}

\author{
T. S. BEZERRA ${ }^{1}$ e A. L. P. S. CAMPOS ${ }^{2}$
}

Universidade Federal do Rio Grande do Norte - UFRN

tiagocentral@ig.com.br ${ }^{1}$ - antonio.luiz@pq.cnpq.br ${ }^{2}$

Artigo submetido em janeiro/2013 e aceito em fevereiro/2014

\section{RESUMO}

Em telecomunicações, com o crescimento da demanda de tráfego de dados nas redes de terceira geração (3G), as operadoras de telefonia móvel têm atentado para o direcionamento dos recursos em infraestrutura nos locais onde se identifica maior necessidade. O direcionamento desses investimentos tem o objetivo de manter a qualidade do serviço prestado, principalmente, em regiões urbanas densas. Neste trabalho, é realizada a predição de séries temporais em redes HSPA WCDMA dos parâmetros: potência recebida (Rx Power), potência de código do sinal recebido (Received Signal
Code Power - RSCP), relação energia por chip em função da interferência (Energy per chip/Interference - Ec/lo) e taxa de transmissão (throughput) na camada física. A coleta dos valores dos parâmetros foi realizada numa rede em pleno funcionamento através de um drive test na cidade de Natal/RN. O modelo utilizado para predição das séries temporais foi o Modelo de Alisamento Exponencial de Holt. $O$ objetivo das predições das séries temporais é verificar para quais parâmetros da rede HSPA - WCDMA o modelo de Holt melhor se adequou.

PALAVRAS-CHAVE: Séries Temporais, Predição, Modelo de Holt, HSPA, WCDMA.

\section{TIME SERIES PREDICTION OF PARAMETERS OF WCDMA NETWORK - HSPA}

\begin{abstract}
In telecommunications, with the growing demand of data traffic in the networks of third generation (3G), the mobile operators have attempted to focus resources on infrastructure in places where it is identified a greater need. The channeling investments have the goal of maintaining quality of service, especially in dense urban areas. In this work, the prediction of time series in WCDMA networks is carried on for the parameters: received power (Rx Power), Received Signal Code Power
\end{abstract}

(RSCP), Energy per chip/Interference (Ec/lo) and throughput at the physical layer. The collection of parameters values was performed on a fully operational network through a drive test in Natal/RN. The model used to predict the time series was the model of Holt exponential smoothing. The objective of the predictions of the series is to check which parameters HSPA network - WCDMA model best adapted Holt. 


\section{INTRODUÇÃO}

A preocupação em prever demandas, manter e melhorar o desempenho dos serviços prestados pela rede tem estimulado estudos de predições para alguns parâmetros nas redes HSPA-WCDMA. Áreas urbanas dos grandes centros brasileiros têm crescido de forma acelerada, com construção de novos prédios, gerando obstruções da cobertura móvel celular e com ocupação concentrada de áreas populosas, o que gera altos índices de congestionamentos comprometendo a qualidade do serviço prestado pelas operadoras de telefonia móvel celular.

Predições de parâmetros de redes móveis têm sido investigadas por diversos autores (CUI, 2010), (KLOCKAR, 2009), (TOUHEED, 2008) e (ZHENG, 2012), sendo que em nenhum destes trabalhos foi realizada predição com modelos de séries temporais para parâmetros da interface aérea em redes HSPA-WCDMA. A maioria dos trabalhos de predição neste tema utiliza simuladores ao invés de medições reais. Investigações teóricas com os mais variados tipos de modelos de predições para séries temporais podem ser encontradas na literatura (WEI, 2006), (MORETTIN, 1981), (MONTGOMERY, 2008), (CHATFIELD, 2000), (BOX, 1994) e (BOX, 1976). A predição para séries temporais de parâmetros da rede HSPA-WCDMA pode ser utilizada pela operadora de telefonia celular como uma ferramenta que objetiva antecipar investimentos de infraestrutura em trechos de cidades que indiquem degradação na rede. Isto é importante porque a operadora pode evitar uma possível percepção negativa do cliente em relação ao serviço prestado por ela, em locais específicos das grandes cidades.

Desta forma, o objetivo principal deste trabalho é realizar predições de parâmetros em rede HSPA-WCDMA para serem utilizadas como um dos itens de suporte, sobre o qual a operadora de telefonia móvel possa tomar decisões em relação ao direcionamento de investimentos de infraestrutura em áreas consideradas críticas em termos de cobertura de radiofrequência. Neste trabalho, foi realizado drive test, em trechos predefinidos, para coleta de amostras de quatro parâmetros da rede HSPA-WCDMA e posterior tratamento utilizando um modelo de série temporal para predições. $O$ modelo utilizado para predição das séries temporais foi o Modelo de Alisamento Exponencial de Holt. A principal contribuição deste trabalho é identificar quais parâmetros de rede coletados do drive test melhor se adequa ao modelo de Holt.

\section{PARÂMETROS DE REDE WCDMA - HSPA}

Existem alguns parâmetros para avaliar o desempenho de uma rede celular móvel HSPA que são os indicadores de desempenho chave (Key Performance Indicator - KPI). Os valores dos KPIs podem ser obtidos por meio das estações rádio base (NodeB) e dos controladores da rede de rádio (RNC - Radio Network Controller) ou pela realização de um drive test. Na seção IV será detalhado o procedimento de drive test adotado neste trabalho.

Durante o drive test são utilizados vários terminais especializados que são capazes de coletar parâmetros de rede durante uma trajetória pré-definida. Os terminais são equipados com um Global Positioning System (GPS), para que se tenha a posição onde cada medição foi realizada. Por exemplo, quando alguns comportamentos estranhos da rede são identificados, pode ser realizado um drive test na área suspeita a fim de obter mais informações sobre as 
condições de funcionamento da rede. No entanto, os drive tests também podem ser executados regularmente para gerar uma base de controle de qualidade da rede sendo utilizado como ferramenta para o processo de otimização da rede. Um drive test realizado com um scanner de frequência pode ser muito útil por ser capaz de capturar as verdadeiras predições de cobertura da rede. É interessante notar que os contadores de rede são capazes de capturar principalmente o comportamento do uplink (HSUPA - High Speed Uplink Packet Acess), ao passo que os relatórios de medição são capazes de coletar o status de downlink (HSDPA - High Speed Uplink Packet Acess). Medições do drive test ilustram principalmente o status do downlink para a trajetória específica do veículo de testes. Portanto, é importante aperfeiçoar essa trajetória do drive test para que ela garanta a aquisição de medições nas áreas onde uma considerável quantidade de tráfego está sendo gerado (SALLENT, 2011).

Neste trabalho, os dados utilizados do drive test foram o Rx Power (nível de sinal na recepção recebido no terminal móvel celular), RSCP (Received Signal Code Power), Ec/lo (Energy per chip/Interference) e a taxa de transmissão (Throughput - Kbps) na camada física (interface aérea).

O Ec/lo é uma relação sinal-interferência onde (E) é a energia média do sinal, (c) é a energia para pontos de potência no tempo em chips e (lo) é a interferência em banda larga e interferência co-canal incluindo a do próprio setor da célula correspondente (NOKIA, 2003). Uma métrica alternativa que pode ser usada para determinar as propriedades de uma célula (BLOGH, 2002) é mostrada na equação (1). Essa expressão calcula a relação sinal-interferência do canal piloto (CPICH) no enlace direto (downlink) e foi proposta na terceira geração de redes celulares da 3GPP Specification.

$\frac{E c}{I o}=\frac{P p i l o t}{\text { Ppilot }+ \text { No }+\sum_{k=1}^{N c e l l s} P_{k} T_{k}}$

equação (1)

em que Ppilot é a potência do canal piloto, $P_{k}$ é a potência total de transmissão da célula $k, T_{k}$ é o ganho da transmissão incluindo o ganho da antena, $N_{0}$ é a densidade espectral de potência para o ruído térmico, para a perda de percurso e para o sombreamento, $N_{\text {cells }}$ é o numero de células da rede.

A camada física da interface de rádio tem sido o tema principal de discussões quando diferentes sistemas celulares são comparados. As estruturas de camada física referem-se diretamente a desempenho ao observar um enlace entre estação terminal móvel e estação rádio base. A camada física tem um grande impacto no que diz respeito à complexidade no processamento da potência da banda base do terminal móvel e estação base (KAARANEN, 2005). $\mathrm{Na}$ terceira geração dos sistemas celulares o foco passa a ser serviços de banda larga, assim, a camada física não mais é utilizada por apenas um único serviço como anteriormente e uma maior flexibilidade é necessária para a introdução dos novos serviços.

A taxa de transferência na camada física depende do número de códigos utilizados, perdas de percursos, ruído e a potencia para tráfego, isso significa que a taxa pode ser variada. De acordo com a equação (2), a taxa de transferência máxima na camada física é de 0,96 Mbps por código HS-PDSCH (High Speed Physical Downlink Shared Channel), sendo 4,8, 9,6 e 14,4 Mbps para 5, 10 e 15 códigos, respectivamente. Na prática, apenas 14 códigos HS-PDSCH são usados para dados, já que, geralmente, dois códigos HS-SCCH (High Speed Shared Control Channel) são reservados para 
sinalização e controle. Assim, a taxa de transferência máxima no nível físico é de 13,44 Mbps (KAARANEN, 2005).

$\mathrm{SF}=\frac{\text { Chip rate }}{\text { Symbol rate }} \Leftrightarrow 16=\frac{3.84}{\frac{\rho_{\mathrm{p}}}{4}}=0.96 \mathrm{Mbps}$

equação (2)

Considerando-se uma taxa de codificação de $75 \%$, a taxa de transferência máxima na camada de controle do enlace de rádio (Radio Link Control - RLC) é de 3,6; 7,2 e 10,08 para 5, 10 e 15 códigos HS-PDSCH, respectivamente. A máxima taxa de transferência permitida no nível de aplicação é de 3,36; 6,72 e 9,4 Mbps para 5, 10 e 15 códigos HS-PDSCH, respectivamente, considerando 93,3\% de 3,6; 7,2 e 10,08, devido à sobrecarga nas camadas MAC (Medium Access Control) e RLC. Na prática, estas taxas de transferências estão entre 3,6 e 8,46 Mbps, considerando-se a razão de erro de bloco (Block Error Ratio - BLER) e aplicações com sobrecargas de $10 \%$. Para usuário único na rede UMTS/HSDPA, apenas o número de códigos HS-PDSCH é o fator limitante que restringe a taxa de transferência máxima disponível para a aplicação (KAARANEN, 2005). A taxa de transmissão coletada pelo drive test realizado para este trabalho é na camada física de uma rede HSPA.

RSCP (Received Signal Code Power) é o nível de potência do código recebido $(\mathrm{dBm})$, refere-se à potência de um código específico entre os símbolos de um piloto recebido (CPICH) e a medição da potência recebida sobre o código. O RSCP é utilizado como parâmetro em conjunto com CPICH para várias funções como handover e a decisão de reseleção de novas células pelo terminal móvel celular na rede WCDMA - HSPA.

RSCP é a soma do RSSI (Received Signal Strength Indicator) com Ec/lo em (dBm), ou seja:

$\operatorname{RSCP}(\mathrm{dBm})=\operatorname{RSSI}(\mathrm{dBm})+\mathrm{Ec} / \mathrm{lo}(\mathrm{dBm})$

equação (3)

Maiores detalhalhes do RSCP podem ser consultados na especificação técnica 3GPP TS 25.215 .

Rx Power é o nível de sinal na recepção do terminal móvel celular recebido da estação móvel celular (NodeB) expressa em dBm. Este parâmetro reflete bem a percepção do cliente no uso dos serviços da rede WCDMA-HSPA.

\section{MODELO DE ALISAMENTO EXPONENCIAL DE HOLT}

Série Temporal é um conjunto de observações ordenadas no tempo (não necessariamente igualmente espaçadas), e que apresentam dependência serial (isto é, dependência entre instantes de tempo). De uma maneira um pouco mais formal, dizemos que uma série temporal é uma realização de um processo estocástico. Na prática, a não ser que estejamos lidando com séries simuladas, a série temporal que está sendo analisada é a única realização disponível do processo estocástico subjacente. Por exemplo, se $Z_{t}$ indica as vendas de sabão em pó de uma marca qualquer no mês $t$, a série temporal $\left\{Z_{1}, Z_{2 \ldots}, Z_{t}\right\}$ que contém os valores efetivamente observados das vendas é a única trajetória disponível do processo estocástico que gerou aquela série (BARROS, 2004).

Um processo estocástico é dito ergódico se uma única realização do processo é o suficiente para caracterizá-lo. Na análise de séries temporais existe apenas uma realização do 
processo disponível e, portanto precisamos supor que o processo subjacente é ergódico, pois iremos usar apenas uma de suas realizações para caracterizá- lo (BARROS, 2004).

Há basicamente, dois aspectos considerados na análise de séries temporais:

- Análise e Modelagem da Série Temporal - descrever a série, verificar suas características mais relevantes e suas possíveis relações com outras séries;

- Previsão da Série Temporal - a partir de valores passados da série, encontrar boas previsões (de curto prazo) de valores futuros da série.

A modelagem de uma série temporal permite mapear toda a estrutura de dependência existente na série. Pode se observar que o resíduo (diferença entre o valor real e o valor da previsão ajustado pelo modelo) não resta nenhuma estrutura, pois já foi coletada pela própria modelagem do modelo. Se o modelo é considerado bom, os resíduos não possuem correlação serial (isto é, correlação entre os resíduos em diferentes instantes de tempo). A modelagem pode explicar um comportamento da série com o menor número de parâmetros o que se pode chamar de parcimônia.

A previsão da série temporal utiliza o histórico dos dados passados para descrever a trajetória da série para o futuro. Os valores passados na maioria das vezes contêm informações relevantes sobre o que ocorrerá no futuro devido à existência da correlação entre as variáveis em diversos instantes de tempo. O conhecimento do passado não quer dizer que será encontrado o valor exato do futuro, mas dar uma boa idéia de quais serão os valores futuros (BARROS, 2004).

Os modelos de alisamento exponencial, como a maioria dos métodos de previsão de séries temporais, se baseiam no princípio que os dados observados no passado contêm informações sobre como a esta série temporal se comportará no futuro (WRIGHT, 1996). As técnicas de alisamento exponencial assumem que valores extremos são causados por ruídos aleatórios e com a suavização das curvas em relação a estes extremos podem ser identificados os padrões básicos do comportamento da série. As grandes vantagens destes métodos são a sua simplicidade, eficiência computacional e a razoável precisão obtida [6].

Existem inúmeras variações de métodos de amortecimento exponencial. Todas elas, na verdade, têm uma característica comum: a informação atual (representada pelo último valor observado) é ponderada com a informação contida nos instantes anteriores. A existência de uma ou mais constantes de amortecimento irá determinar como funciona este mecanismo de ponderação ou, em outras palavras, o quão rapidamente decai a influência das observações passadas. Os parâmetros do modelo sofrem uma atualização sequencial, e a chegada de uma nova observação resulta numa nova estimativa dos parâmetros (BARROS, 2004).

O modelo de Holt é mais adequado para séries temporais que há uma tendência linear e não existe sazonalidade. Os seus parâmetros de alisamento são os de nível e de tendência da série. O modelo de Holt é representado pelas equações (4), (5) e (6) (BARROS, 2004):

$M_{\mathrm{t}}=\alpha \mathrm{Z}_{\mathrm{t}}+(1-\alpha) \cdot\left(M_{\mathrm{t}-1}+\hat{a}_{2}(T-1)\right)$

equação (4)

$\hat{a}_{2}(T)=\beta \cdot\left(M_{\mathrm{t}}-M_{\mathrm{t}-1}\right)+(1-\beta) \cdot \hat{a}_{2}(T-1)$

equação (5)

$\hat{a}_{1}(T)=M_{\mathrm{t}}$

equação (6) 
em que: $\alpha$ e $\beta$ são as constantes de amortecimento de nível e tendência respectivamente, os valores das constantes variam entre 0 e $1 ; M_{\mathrm{t}}$ é a estimativa do nível da série; e $\hat{a}_{2}$ é a taxa de tendência (crescimento) estimada.

As constantes de amortecimento usadas para cálculo do nível e tendência são diferentes. $\mathrm{Na}$ equação de atualização do nível, o novo nível é função de três variáveis: a observação mais recente $\left(\mathrm{Z}_{\mathrm{t}}\right)$, a estimativa anterior do nível $\left(M_{\mathrm{t}-1}\right)$ e a taxa de crescimento estimada no instante anterior $\left(\hat{a}_{2}(T-1)\right)$. A taxa de crescimento atualizada é função da taxa de crescimento no instante anterior e também depende da diferença entre os níveis nos instantes $T-1$ e $T$ (BARROS, 2004).

Assim como ocorre no modelo de alisamento simples, é necessário ter valores iniciais dos parâmetros para que se possa dar início à série prevista. Os valores iniciais podem ser estimados pelas equações (7) e (8) (MAKRIDAKIS, 1998).

$$
\begin{array}{ll}
\hat{a}_{1}(1)=Z_{1} & \text { equação (7) } \\
\hat{a}_{2}(1)=\frac{\left(Z_{2}-Z_{1}\right)+\left(Z_{4}-Z_{3}\right)}{2} & \text { equação (8) }
\end{array}
$$

\section{MEDIÇÃO DOS PARÂMETROS EM DRIVE TEST}

As medições dos parâmetros foram realizadas através de um drive test numa rede HSPAWCDMA em pleno funcionamento na cidade de Natal - RN nos bairros de Lagoa Nova, Nossa Senhora de Nazaré, Quintas, Ribeiras e bairros adjacentes como mostra a Figura 1. Os equipamentos utilizados no drive test foram um aparelho celular do fabricante LG modelo Optimus ME P350 para chamadas de voz, um aparelho celular e modem Motorola RAZR HD XT925 para trafegar dados em HSPA e um Scanner DRT4301A GSM/WCDMA (806/2500 MHz) modelo MPLVDB800/1900S para coleta de dados de parâmetros oriundos da rede WCDMA/HSPA. Também foi utilizado o software comercial X-CAL versão D3.3.1.50, da Empresa Accuver e um servidor FTP (File Transfer Protocol) para execução de downloads de arquivos. 0 software é uma solução baseada em tempo real para medição de desempenho e otimização de redes sem fio. O XCAL coleta mensagens das camadas 1, 2, 3 do modelo OSI e pacotes TCP/IP. As coletas são efetuadas na interface aérea e também na interface de dados em todas as tecnologias comercialmente disponíveis (CDMA IS95A/B, 1XRTT, EVDO (Rev. 0 e Rev. A), GSM, GPRS, EDGE, UMTS, HSDPA e HSUPA). A Tabela 1 exibe a legenda para os níveis de sinal (Rx Power) do drive test mostrado na Figura1.

Tabela 1 - Legenda dos níveis de sinal do drive test.

\begin{tabular}{l|l}
\hline LEGENDA & \multicolumn{1}{|c}{ Rx POWER $(\mathrm{dB})$} \\
\hline & $-200<=$ Rx Power $(\mathrm{dBm})<-$ \\
& 100 \\
& $-100<=$ Rx Power $(\mathrm{dBm})<-$ \\
90 & $-90<=$ Rx Power $(\mathrm{dBm})<-80$ \\
\hline & $-80<=$ Rx Power $(\mathrm{dBm})<-60$ \\
\hline & $-60<=$ Rx Power $(\mathrm{dBm})<-$ \\
& 100 \\
\hline
\end{tabular}




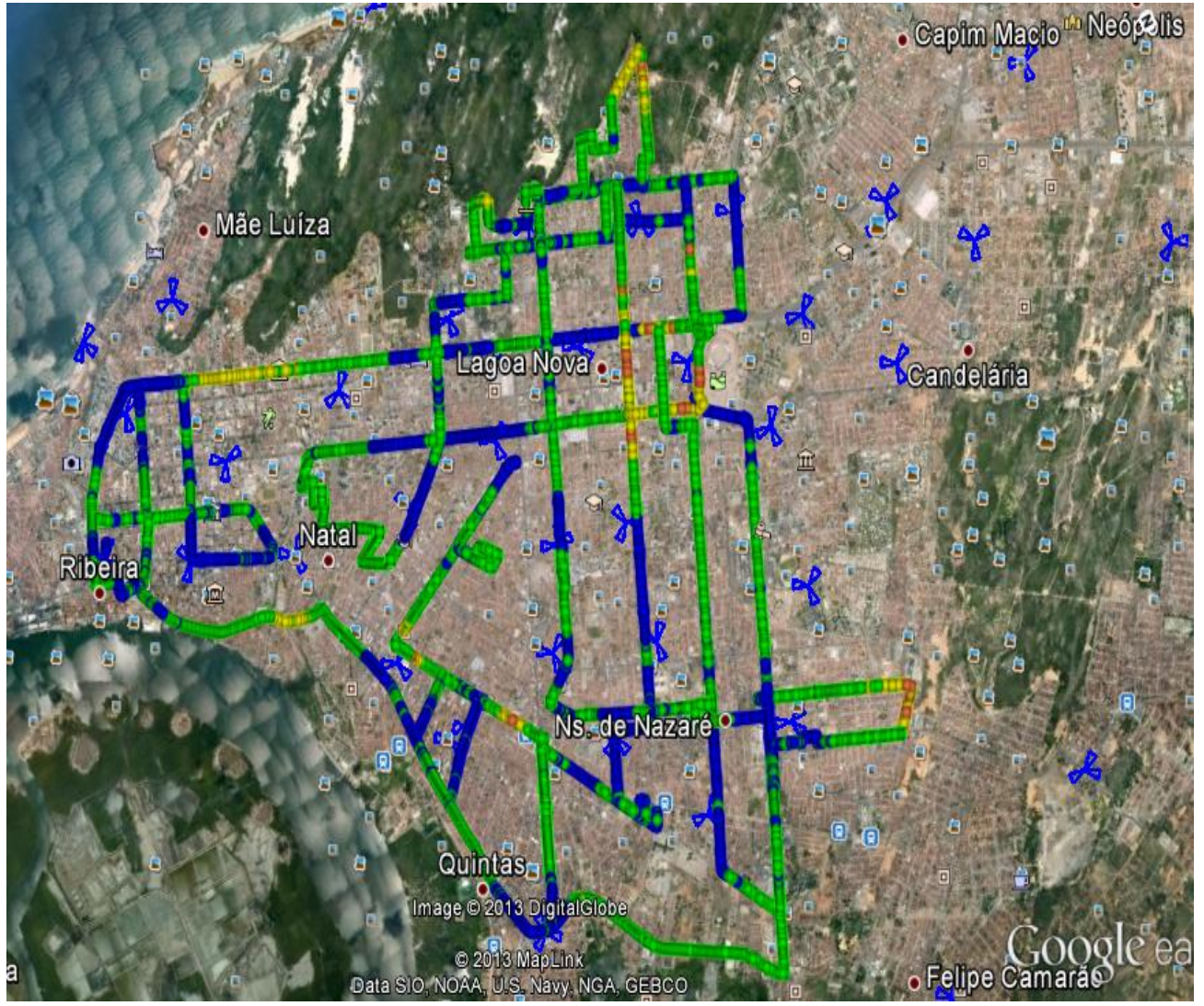

Figura 1 - Mapa mostrando o drive test realizado em Natal.

A coleta dos valores dos parâmetros foi realizada com auxílio de um veículo automotivo em deslocamento, com a velocidade média em torno de $21,13 \mathrm{Km} / \mathrm{h}$ no dia 17/09/2013 entre os horários de $09 \mathrm{~h} 40 \mathrm{~min} 47 \mathrm{seg}$ e $13 \mathrm{~h} 16 \mathrm{~min} 58 \mathrm{seg}$ num de total $3 \mathrm{~h} 36 \mathrm{~min} 11 \mathrm{seg}$. A cada segundo foi realizado uma amostra de medição dos parâmetros com um total de 12.971 amostras coletadas.

\section{ANÁLISE DAS SÉRIES TEMPORAIS DOS PARÂMETROS ANALISADOS}

Para manipulação dos dados e melhor visualização dos gráficos, foram separadas 2880 (22,20\% do total) amostras e segmentadas em onze séries de para análise, modelagem e predição utilizando o modelo de Alisamento Exponencial de Holt conforme Tabela 1.

Para avaliação do modelo das séries foram utilizadas as medidas de acurácia que são estatísticas que traduzem numericamente a precisão de um dado modelo. Por possuírem esta propriedade, estas medidas são utilizadas na construção dos modelos, de modo que sejam minimizadas, ou então na escolha de um modelo dentro de um grupo de modelos disponíveis (ARMSTRONG, 1992). Para ter um controle destes erros é importante que se defina uma função de perda (MORETTIN, 1981). Com o passar do tempo, o uso dos métodos absolutos, o MAD (Mean Absolute Deviation) equação (8) e o MSD (Mean Squared Deviation) equação (9) têm sido os mais populares devido as suas relevâncias teóricas no modelamento estatístico (HYNDMAN, 2006). Já dentre as medidas relativas, a mais utilizada é o MAPE (Mean Absolute Percentage Error), equação (10), pois esta medida é bem interpretativa e muito apropriada no planejamento (FADER, 2003). 
No Minitab foram calculadas as três medidas de acurácia do modelo ajustado: MAPE, MAD e MSD para cada uma das previsões do modelo de Alisamento Exponencial de Holt. Para todas as três medidas, quanto menor o valor, melhor o ajuste do modelo. Foram utilizadas essas estatísticas para comparar os ajustes dos modelos das diferentes amostras. Neste trabalho, caso haja o empate na escolha da melhor série, por exemplo, três séries distintas cada uma com apenas uma medida de acurácia com menor valor dentre as demais, a série que tiver o menor MAPE será escolhida para ser realizada a predição. O MAPE é uma medida interpretativa e mais apropriada para realização de planejamentos (FADER, 2003).

$$
\begin{aligned}
& M A D=\frac{\sum_{t=1}^{n} \frac{\mathrm{z}_{\mathrm{t}}-\widehat{\mathrm{z}}_{\mathrm{t}}}{\mathrm{z}_{\mathrm{t}}}}{n} \\
& M S D=\frac{\sum_{t=1}^{n}\left(\frac{\mathrm{z}_{\mathrm{t}}-\widehat{\mathrm{z}}_{\mathrm{t}}}{\mathrm{z}_{\mathrm{t}}}\right)^{2}}{n \widehat{\mathrm{z}}_{\mathrm{t}}} \\
& M A P E=\frac{\sum \frac{\mathrm{z}_{\mathrm{t}}-\mathrm{z}_{\mathrm{t}}}{n}}{\mathrm{z}_{\mathrm{t}}} \cdot 100,\left(\mathrm{Z}_{\mathrm{t}} \neq 0\right)
\end{aligned}
$$$$
\text { equação (9) }
$$$$
\text { equação (10) }
$$$$
\text { equação (11) }
$$

em que, $Z_{t}$ é o valor atual da série temporal, $\widehat{Z_{t}}$ valor atual previsto da série e $n$ é o número de observações.

$\mathrm{Na}$ Tabela 2 constam os dados da modelagem com o modelo de Alisamento Exponencial Duplo de Holt do parâmetro Rx Power utilizando o software estatístico Minitab ${ }^{\circledR}$ 16.2.2.0 - (C) 2010 Minitab Inc. Os valores de $\alpha$ (constante de amortecimento de nível) e $\beta$ (constante de amortecimento de tendência) são estimados durante o ajuste dos modelos pelo próprio software Minitab Inc.

Tabela 2 - Índices do Modelo de Holt para o parâmetro Rx Power.

\begin{tabular}{c|c|c|c|c|c|c}
\hline ÍNDICE & AMOSTRAS & $\alpha$ & $\beta$ & MAPE & MAD & MSD \\
\hline 1 & 250 & 1,1268 & 0,0045 & $\mathbf{3 , 4 9 1 2}$ & $\mathbf{2 , 3 7 9 2}$ & $\mathbf{1 1 , 1 0 3 1}$ \\
\hline 2 & 230 & 1,1006 & 0,0200 & 4,6204 & 2,7867 & 15,943 \\
\hline 3 & 300 & 1,0849 & 0,0095 & 4,5799 & 2,9288 & 15,7957 \\
\hline 4 & 250 & 1,0947 & 0,0085 & 3,8204 & 2,6004 & 15,1365 \\
\hline 5 & 250 & 1,0750 & 0,0135 & 6,4174 & 3,6918 & 24,1871 \\
\hline 6 & 250 & 1,1476 & 0,0068 & 4,9942 & 3,3087 & 18,9706 \\
\hline 7 & 250 & 1,1157 & 0,1559 & 5,0588 & 3,5542 & 20,2150 \\
\hline 8 & 290 & 1,0642 & 0,0123 & 5,2828 & 3,2162 & 17,6230 \\
\hline 9 & 270 & 1,1709 & 0,0103 & 6,1035 & 3,3558 & 20,8544 \\
\hline 10 & 250 & 1,1859 & 0,0106 & 6,2310 & 3,8643 & 22,8321 \\
\hline 11 & 280 & 1,1554 & 0,0111 & 6,6479 & 3,7456 & 24,6944 \\
\hline
\end{tabular}

A série temporal de número um das amostras Rx Power foi a escolhida para análise estatística e predição devido às medidas de precisão (MAPE, MAD e MSD) terem os menores valores comparados com as medidas de acurácia das outras amostras conforme apresentado na Tabela 2.

A série temporal das amostras Rx Power_1 se caracteriza pela estatítica descritiva apresentada na Tabela 3 e figuras 2 e 3. Para essa série, pode-se observar que a potência média do sinal recebido foi de $-70 \mathrm{dBm}$, o que mostra que essa região de cobertura está satisfatória, uma vez que o limiar dos celulares é em torno de $-100 \mathrm{dBm}$. A variância foi de 16,77, o que confirma a natureza variável do ambiente de propagação. Os níveis mínmo e máximo da potência recebida foram $-77,17 \mathrm{dBm}$ e $-55,17 \mathrm{dBm}$, mostrando a boa cobertura da rota considerada. 
Tabela 3 - Estatística descritiva para o parâmetro Rx Power.

\begin{tabular}{c|c|c|c|c|c|c|c}
\hline VARIÁVEL & MÉDIA & DESVIO PADRÃO & VARIÂNCIA & VALOR MÍNIMO & VALOR MÁXIMO & ASSIMETRIA & CURTOSE \\
\hline Rx Power & $-70,19$ & 4,09 & 16,77 & $-77,17$ & $-55,17$ & 1,4 & 1,99 \\
\hline
\end{tabular}

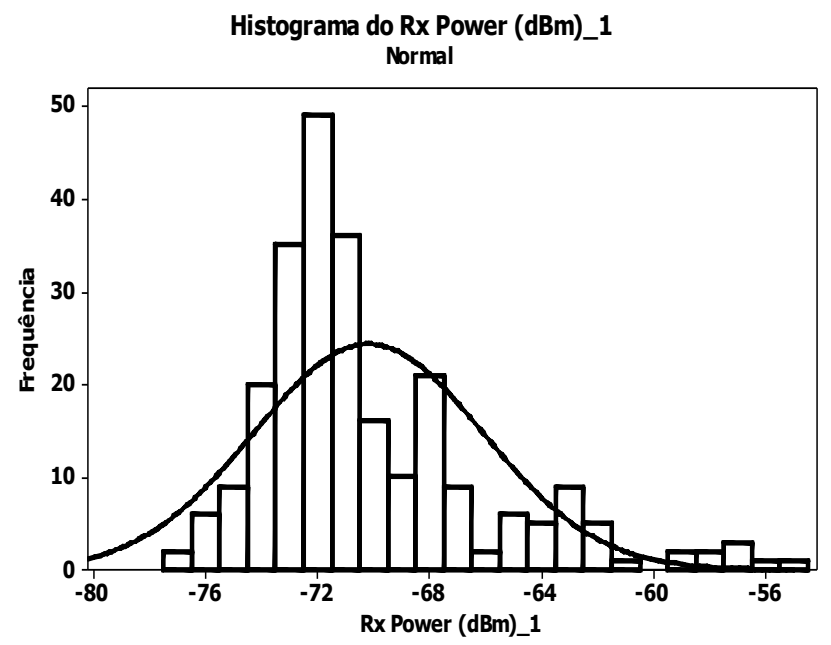

Figura 2 - Histograma do parâmetro Rx Power.

Autocorrelação Rx Power (dBm) 1 (com limite de significância de 5\% na autocorrelação)

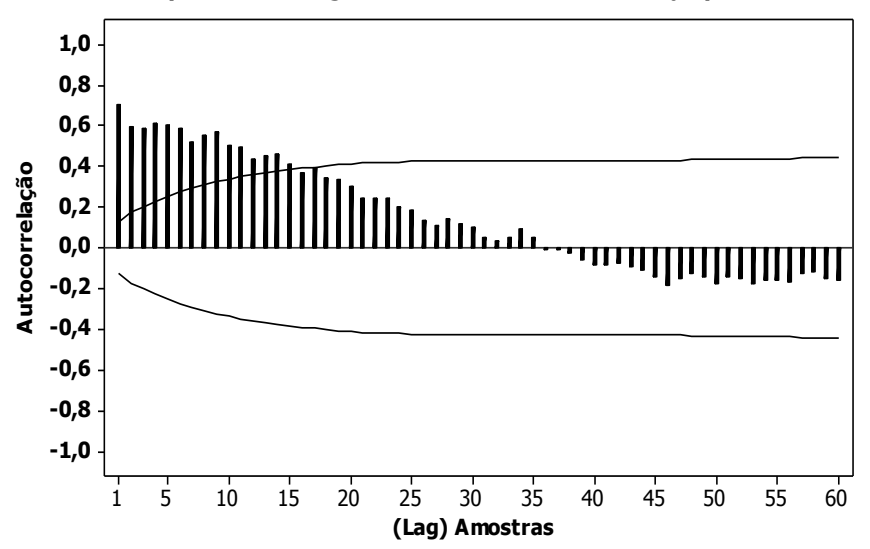

Figura 3 - Autocorrelação do parâmetro Rx Power.

A predição de $10 \%(25)$ do volume de amostras (250) da série temporal Rx Power_1 é apresentada na Figura 4. 


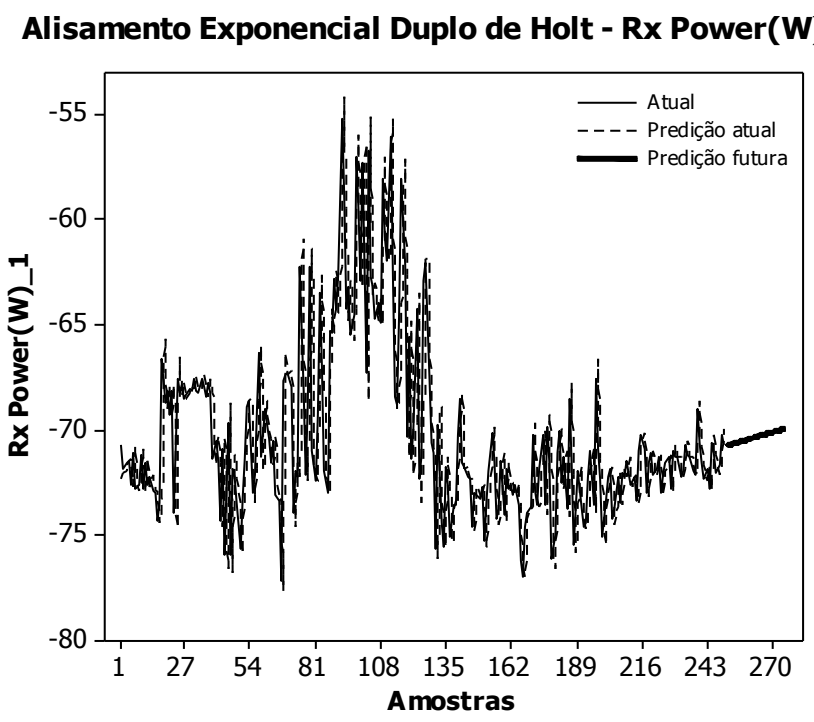

Figura 4 - Predição para Rx Power_1 usando o Modelo de Holt.

Os dados da análise e modelagem da série temporal do parâmetro $\operatorname{RSCP}(\mathrm{dBm})$ são apresentados Tabela 4. A série temporal de número um das amostras RSCP foi a escolhida para análise estatística e predição devido as medidas de acurácia terem os menores valores comparados com as medidas de acurácia das outras amostras conforme apresentado Tabela 4.

Tabela 4 - Índices do Modelo de Holt para o parâmetro RSCP.

\begin{tabular}{c|c|c|c|c|c|c}
\hline ÍNDICE & AMOSTRAS & $\alpha$ & $\beta$ & MAPE & MAD & MSD \\
\hline 1 & 250 & 1,9819 & 0,0125 & $\mathbf{2 , 4 6 2 7}$ & $\mathbf{1 , 8 5 4 1}$ & $\mathbf{6 , 1 8 4 2}$ \\
\hline 2 & 250 & 1,1172 & 0,0163 & 3,5826 & 2,3745 & 10,2858 \\
\hline 3 & 300 & 1,1329 & 0,0088 & 3,1736 & 2,3194 & 9,5222 \\
\hline 4 & 270 & 1,2398 & 0,0066 & 3,0201 & 2,2703 & 10,6503 \\
\hline 5 & 300 & 1,1845 & 0,0092 & 2,8443 & 2,9879 & 15,7612 \\
\hline 6 & 250 & 1,1487 & 0,0098 & 2,7088 & 2,3214 & 9,7116 \\
\hline 7 & 280 & 1,1008 & 0,0122 & 3,6656 & 2,6617 & 12,1464 \\
\hline 8 & 250 & 1,2085 & 0,0056 & 5,1168 & 3,3376 & 18,2620 \\
\hline 9 & 280 & 1,1493 & 0,0133 & 3,7110 & 2,5225 & 8,7718 \\
\hline 10 & 230 & 1,1513 & 0,0132 & 4,4077 & 3,1308 & 16,6547 \\
\hline 11 & 219 & 1,2593 & 0,0003 & 4,6406 & 2,8463 & 13,7329 \\
\hline
\end{tabular}

Tabela 5 - Estatística descritiva para o parâmetro RSCP.

\begin{tabular}{c|c|c|c|c|c|c|c}
\hline VARIÁVEL & MÉDIA & DESVIO PADRÃO & VARIÂNCIA & VALOR MÍNIMO & VALOR MÁXIMO & ASSIMETRIA & CURTOSE \\
\hline RSCP & $-77,39$ & 5,04 & 25,42 & $-84,47$ & $-60,84$ & 1,17 & 1,10 \\
\hline
\end{tabular}

A série temporal das amostras RSCP_1 se caracteriza pela estatítica descritiva apresentada na Tabela 5 e figuras 5 e 6 . Para essa série, pode-se observar que a potência média de código recebida foi de $-77,39 \mathrm{dBm}$. A variância foi de 25,42 , o que confirma a natureza variável do ambiente de propagação. Os níveis mínmo e máximo da potência recebida foram $84,47 \mathrm{dBm}$ e $-60,84 \mathrm{dBm}$, mostrando a boa cobertura da rota considerada. A predição de $10 \%$ (25) do volume de amostras (250) da série temporal Rx Power_1 é apresentada na Figura 4. 


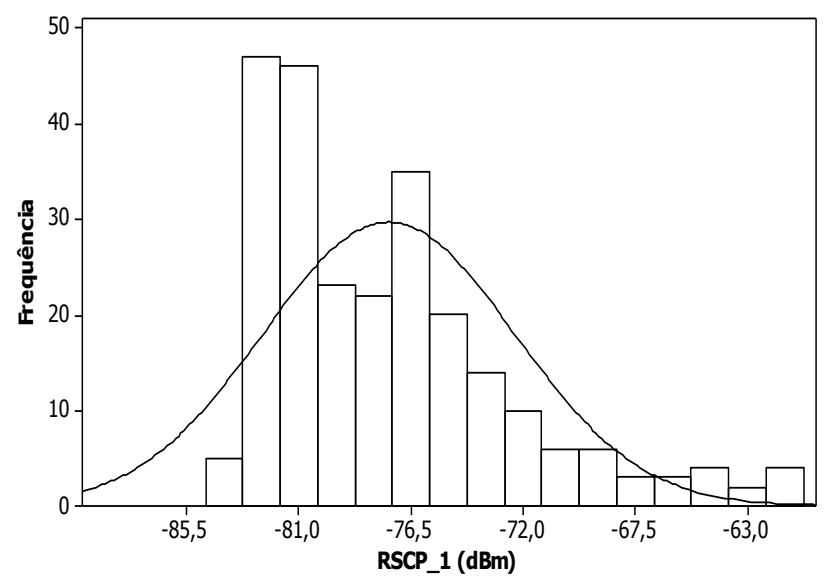

Figura 5 - Histograma do parâmetro RSCP.

Função de Autocorrelação - RSCP_1 (dBm)

(Com limite de significância de $5 \%$ na autocorrelação)

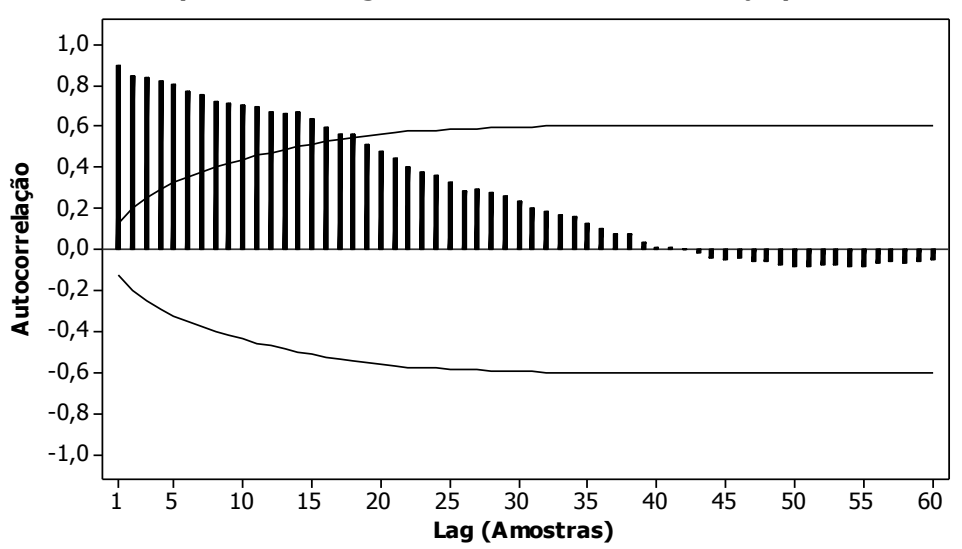

Figura 6 - Autocorrelação do parâmetro RSCP.

A predição de $10 \%$ (25) do volume de amostras (250) da série temporal RSCP_1 (dBm) é apresentada na Figura 7. Os dados da análise e modelagem da série temporal do parâmetro Ec/lo são listados na Tabela 6 . A série temporal de número um das amostras Ec/lo tem o menor MAPE, a amostra de número quatro tem menor MAD e a amostra de número nove tem o menor MSD. A série de número um um foi escolhida para análise estatística e predição pois a medida de acurácia MAPE foi definida como critério de desempate na escolha das amostras. 


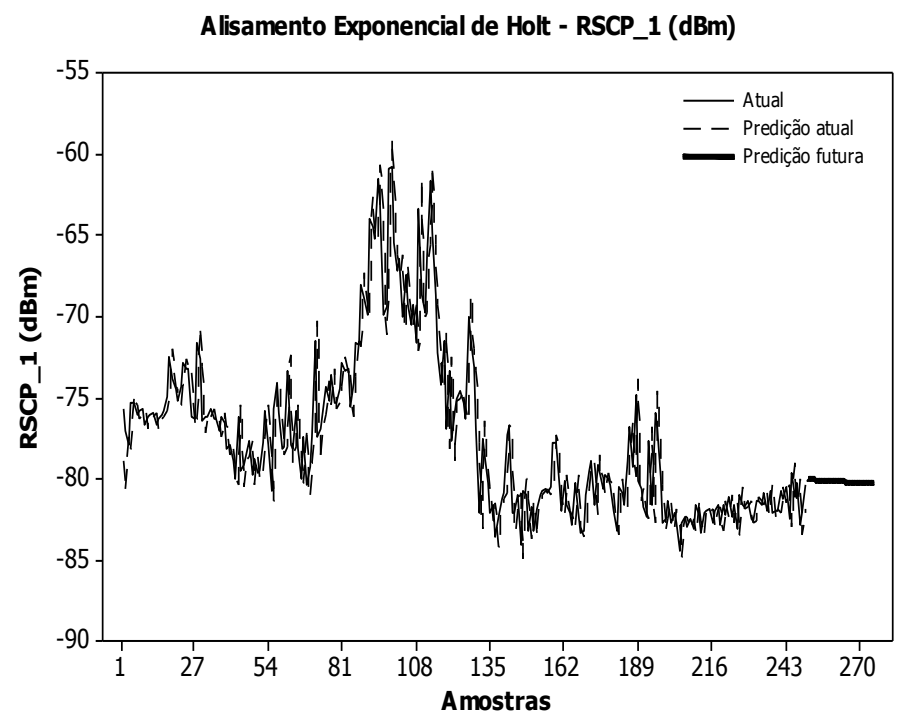

Figura 7 - Predição para RSCP_1 usando o Modelo de Holt.

Tabela 6 - Índices do Modelo de Holt para o parâmetro Ec/lo.

\begin{tabular}{c|c|c|c|c|c|c}
\hline ÍNDICE & AMOSTRAS & $\alpha$ & $\beta$ & MAPE & MAD & MSD \\
\hline 1 & 245 & 1,1230 & 0,0053 & $\mathbf{1 7 , 6 8 0 5}$ & 1,0775 & 2,2854 \\
\hline 2 & 290 & 1,0966 & 0,0114 & 32,5205 & 1,4583 & 3,8248 \\
\hline 3 & 220 & 1,1235 & 0,0091 & 26,2244 & 1,5698 & 4,6733 \\
\hline 4 & 250 & 1,2281 & 0,0096 & 17,994 & $\mathbf{1 , 0 3 2 8}$ & 2,2163 \\
\hline 5 & 270 & 1,0968 & 0,0129 & 32,1677 & 1,4699 & 4,2776 \\
\hline 6 & 300 & 1,1803 & 0,0074 & 27,4336 & 1,4220 & 4,2751 \\
\hline 7 & 250 & 1,1538 & 0,0087 & 34,5699 & 1,4503 & 4,8225 \\
\hline 8 & 250 & 1,1414 & 0,0144 & 26,4966 & 1,5040 & 4,8268 \\
\hline 9 & 300 & 1,2177 & 0,0059 & 18,3014 & 1,0560 & $\mathbf{2 , 1 1 7 3}$ \\
\hline 10 & 250 & 1,1773 & 0,0037 & 28,3986 & 1,5874 & 4,7857 \\
\hline 11 & 254 & 1,0832 & 0,0108 & 20,4163 & 1,1153 & 3,0124 \\
\hline
\end{tabular}

A série temporal das amostras Ec/lo_1 se caracteriza pela estatítica descritiva apresentada na Tabela 7 e figuras 8 e 9. Para essa série, pode-se observar que a relação sinalinterferência média foi de $-7,314 \mathrm{dBm}$. A variância, nesse caso, foi mais baixa, sendo de 4,738. Os níveis mínmo e máximo do Ec/lo foram - 11,02 dBm e-2,64 dBm, respectivamente.

Tabela 7 - Estatística descritiva para o parâmetro Ec/lo.

\begin{tabular}{c|c|c|c|c|c|c|c}
\hline VARIÁVEL & MÉDIA & DESVIO PADRÃO & VARIÂNCIA & VALOR MÍNIMO & VALOR MÁXIMO & ASSIMETRIA & CURTOSE \\
\hline Ec/lo & $-7,314$ & 2,177 & 4,738 & $-11,020$ & $-2,640$ & 0,40 & $-0,97$ \\
\hline
\end{tabular}




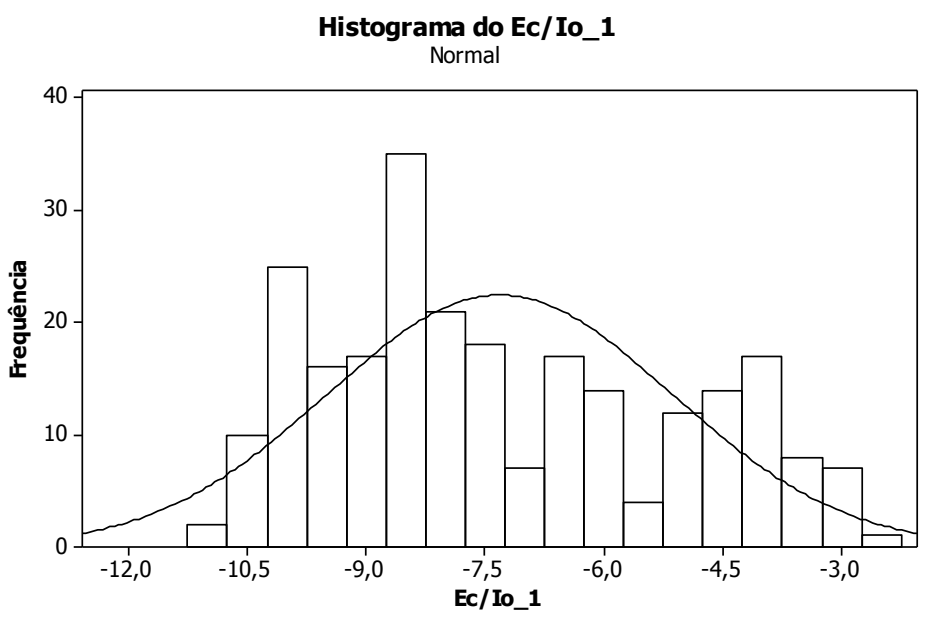

Figura 8 - Histograma do parâmetro Ec/lo.

Função de Autocorrelação - Ec/Io_1 (Com limite de significância de 5\% na autocorrelação)

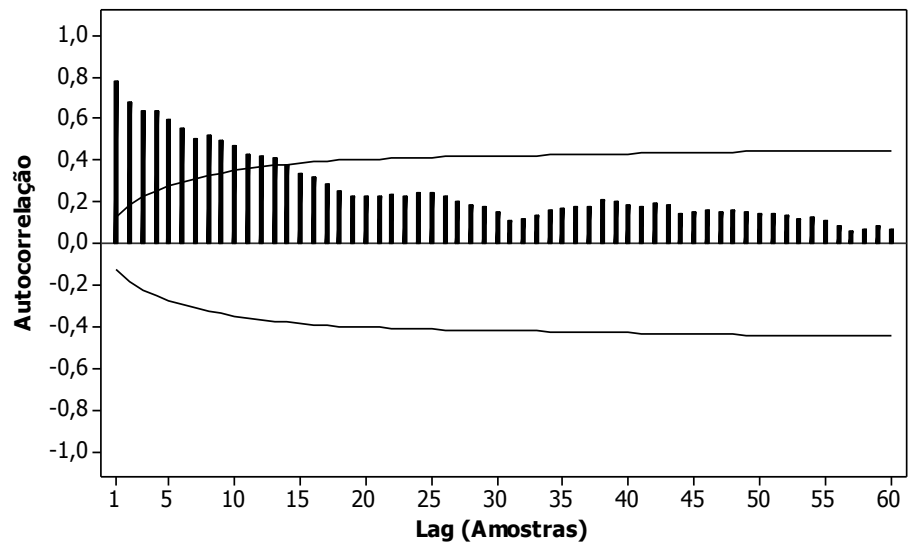

Figura 9 - Autocorrelação do parâmetro Ec/lo.

A predição de $10 \%$ (25) do volume de amostras (250) da série temporal Ec/lo_1 é apresentada na Figura 10. Os dados da análise e modelagem da série temporal do parâmetro Ec/lo na Tabela 8. A série temporal de número um das amostras Throughput tem o menor valor do MAPE, mas a amostra de numero quatro tem menor valor de MAD e MSD. A amostra quatro foi a escolhida para análise estatística e predição por ter apresentado duas medidas de acurácia de menor valor conforme apresentado na Tabela 8. 


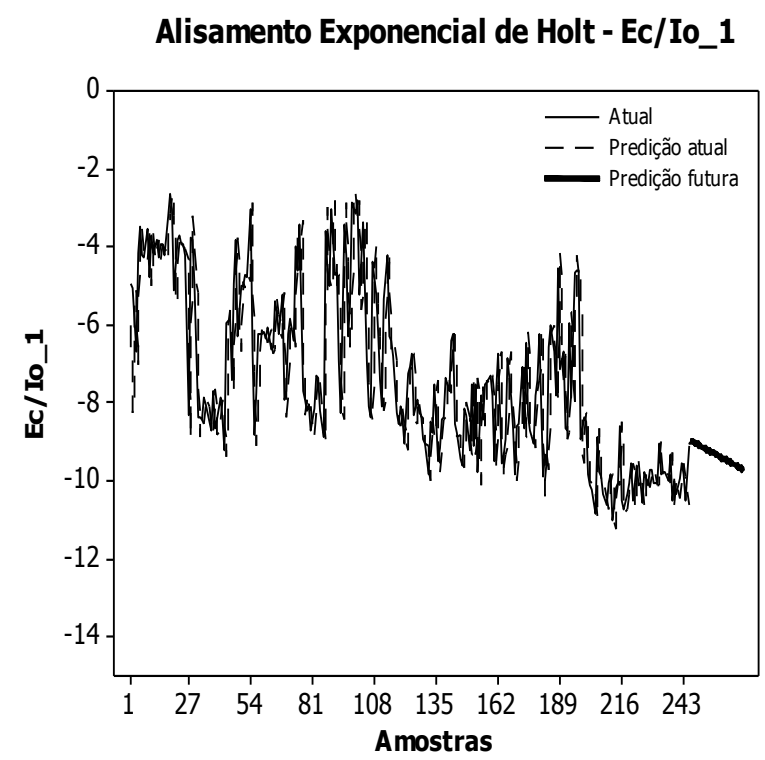

Figura 10 - Predição para Ec/lo_1 usando o Modelo de Holt.

Tabela 8 - Índices do Modelo de Holt para o parâmetro Throughput.

\begin{tabular}{c|c|c|c|c|c|c}
\hline ÍNDICE & AMOSTRAS & $\alpha$ & $\beta$ & MAPE & MAD & MSD \\
\hline 1 & 230 & 1,120 & 0,008 & 53 & 820 & 1278468 \\
\hline 2 & 250 & 0,976 & 0,017 & 152 & 782 & 1697467 \\
\hline 3 & 240 & 1,041 & 0,007 & 163 & 1049 & 2545927 \\
\hline 4 & 250 & 1,242 & 0,010 & 88 & $\mathbf{3 7 0}$ & $\mathbf{4 6 7 6 8 6}$ \\
\hline 5 & 300 & 1,190 & 0,003 & 65 & 941 & 1890325 \\
\hline 6 & 260 & 1,080 & 0,001 & 322 & 916 & 1811863 \\
\hline 7 & 300 & 1,151 & 0,006 & 1067 & 1081 & 2987919 \\
\hline 8 & 240 & 1,1972 & 0,010 & 853 & 987 & 2036691 \\
\hline 9 & 200 & 1,1327 & 0,012 & 290 & 1089 & 2053908 \\
\hline 10 & 300 & 1,1763 & 0,006 & 153 & 696 & 975151 \\
\hline 11 & 299 & 1,0169 & 0,014 & 934 & 1218 & 3126556 \\
\hline
\end{tabular}

A série temporal das amostras Throughput_4 se caracteriza pela estatítica descritiva apresentada na Tabela 9 e figuras 11 e 12. Para essa série, pode-se observar que a taxa de transmissão média foi de 1001,1 Kbps. A variância, nesse caso, foi muito alta, o que mostra que esse tipo de tecnologia não é muito apropriada para alguns tipos de aplicações. A taxa de transmissão máxima foi de 6190,6 Kbps.

Tabela 9 - Estatística descritiva para o parâmetro Throughput.

\begin{tabular}{c|c|c|c|c|c|c|c}
\hline VARIÁVEL & MÉDIA & DESVIO PADRÃO & VARIÂNCIA & VALOR MÍNIMO & VALOR MÁXIMO & ASSIMETRIA & CURTOSE \\
\hline Throughput & 1001,1 & 1473,1 & 2169914,1 & 0,0 & 6190,6 & 1,62 & 1,94 \\
\hline
\end{tabular}




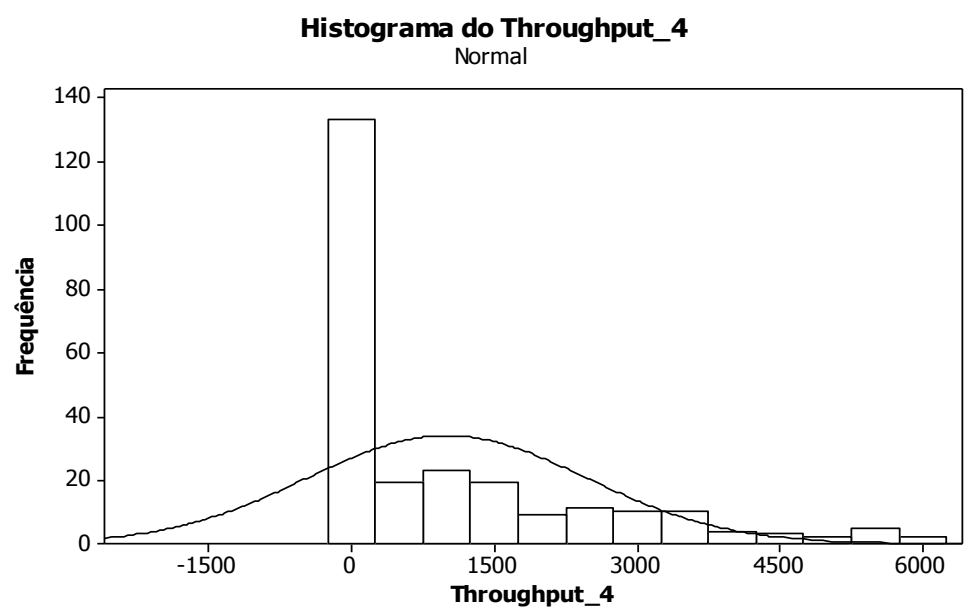

Figura 11 - Histograma do parâmetro throughput.

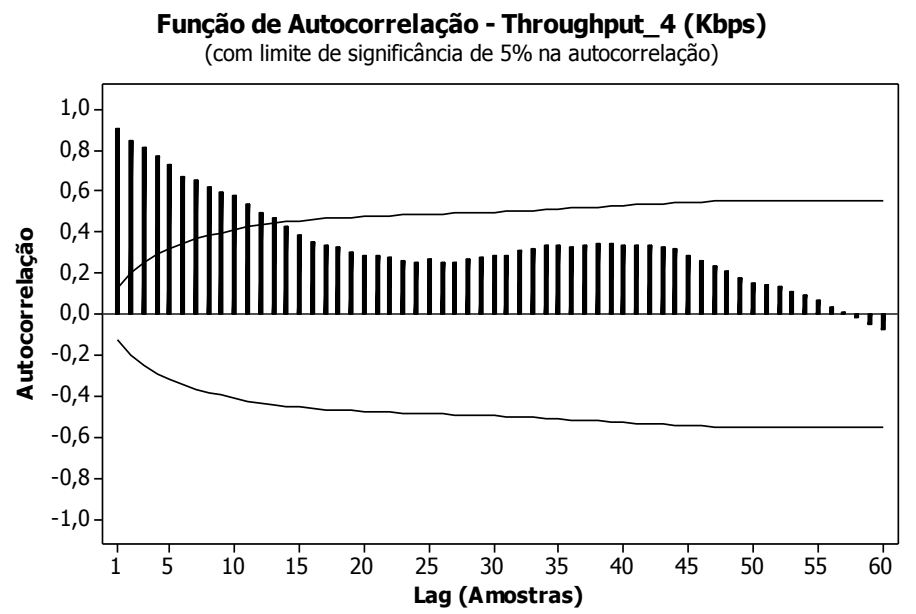

Figura 12 - Autocorrelação do parâmetro throughput.

Pode-se ver na Tabela 9 um valor muito elevado para o desvio padrão e, consequentemente, para a variância. Isso ocorre por que o throughoput é medido na camada física, sendo a taxa de velocidade de download na recepção do aparelho móvel usado no drive test. Por se tratar de tráfego por rajada, essa taxa depende das solicitações do dispositivo móvel e tem momentos em que a rede HSDPA não transmite para o móvel por diferentes motivos. Tem momentos em que essa taxa é zero e de repente vai para $5000 \mathrm{Kpbs}$. Então, muitas vezes o desvio da média é alto entre uma amostra e outra.

A predição de $10 \%$ (25) do volume de amostras (250) da série temporal throughput_4 é apresentada na Figura 13. 


\section{Alisamento Exponencial de Hollt - Throughput_4}

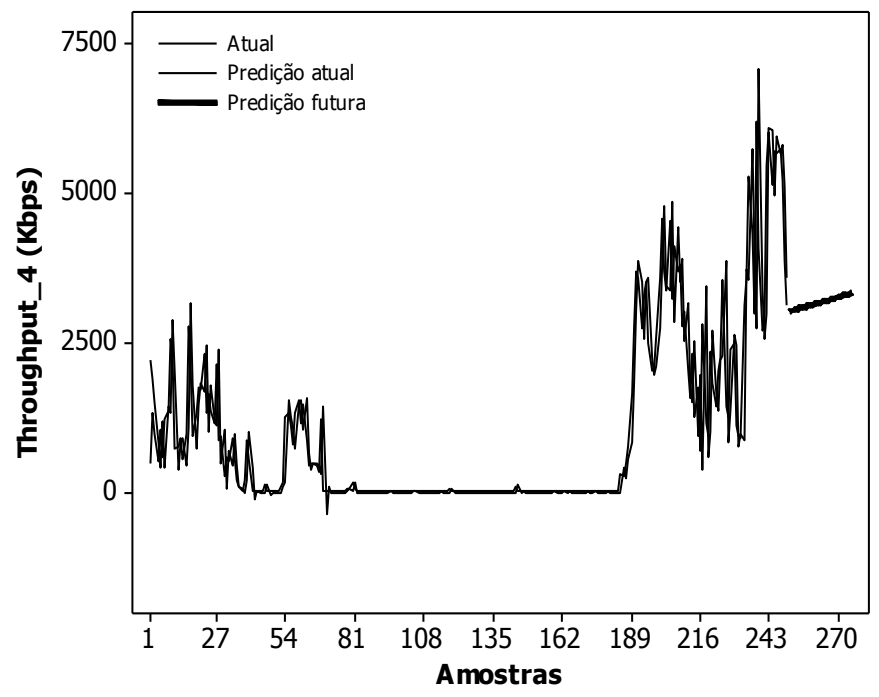

Figura 13 - Predição para throughput_1 usando o Modelo de Holt.

\section{RESULTADOS DE PREDIÇÃO DAS SÉRIES TEMPORAIS DOS PARÂMETROS ANALISADOS}

Comparando as quatro séries trabalhadas na sessão anterior, o modelo ajustado de Alisamento Exponencial de Holt das séries temporais que apresentou o menor MAPE foi a RSCP_1, o menor MAD e MSD foi à série temporal Ec/lo_1 conforme listado na Tabela 10.

Foram calculadas as médias das constantes de nível $\alpha$ e tendência $\beta$ das tabelas IV e VI dos parâmetros RSCP e Ec/lo para análise e modelagem de um novo modelo de Alisamento Exponencial de Holt obtendo novas medidas de acurácia para comparar com as medidas de acurácia de amostras externas que foram obtidas em novo drive test, distinto do drive test apresentado neste trabalho. Na Tabela 11 são apresentadas as medidas de acurácia da média das constantes de tendência e nível, na Tabela 12 são apresentadas as medidas de acurácia das amostras externas (novo drive test).

Tabela 10 - Resultados finais para o Modelo de Holt.

\begin{tabular}{c|c|c|c|c|c|c}
\hline PARÂMETRO & AMOSTRAS & $\alpha$ & $\beta$ & MAPE & MAD & MSD \\
\hline Rx Power_1 & 250 & 1,1268 & 0,0045 & 3,4912 & 2,3792 & 11,1031 \\
\hline RSCP_1 & 250 & 1,9819 & 0,0125 & 2,4627 & 1,8541 & 6,1842 \\
\hline Ec/lo_1 & 245 & 1,1230 & 0,0053 & 17,680 & 1,0775 & 2,2854 \\
\hline Throughput_4 & 250 & 1,2420 & 0,01036 & 88 & 370 & 467686 \\
\hline Rx Power_1 & 250 & 1,1268 & 0,0045 & 3,4912 & 2,3792 & 11,1031 \\
\hline
\end{tabular}


Tabela 11 - Modelo de Holt - Médias das constantes $\alpha$ e $\beta$.

\begin{tabular}{c|c|c|c|c|c|c}
\hline PARÂMETRO & AMOSTRAS & $\alpha$ & $\beta$ & MAPE & MAD & MSD \\
\hline RSCP & 250 & 1,2431 & 0,0098 & 2,4868 & 1,8728 & 6,3261 \\
\hline Ec/lo & 245 & 1,1474 & 0,0090 & 17,5990 & 1,0793 & 2,2896 \\
\hline
\end{tabular}

Tabela 12 - Modelo de Holt - novo drive test.

\begin{tabular}{c|c|c|c|c|c|c}
\hline PARÂMETRO & AMOSTRAS & $\alpha$ & $\beta$ & MAPE & MAD & MSD \\
\hline RSCP & 250 & 0,990 & 0,009 & $\mathbf{2 , 4 4 7 2}$ & 2,0268 & 7,4072 \\
\hline Ec/lo & 245 & 1,1082 & 0,0096 & 13,1111 & $\mathbf{0 , 9 8 0 7}$ & $\mathbf{2 , 2 8 5 5}$ \\
\hline
\end{tabular}

Comparando as séries temporais que constam as médias constantes de amortecimento de nível e tendência na Tabela 10 e as amostras do novo drive test na Tabela 11, foi observado que o parâmetro RSCP apresentou o melhor MAPE e o parâmetro Ec/lo apresentou os melhores MAD e MSD ambos oriundos do novo drive test. Diante dos resultados apresentados, será realizado o estudo das duas séries a seguir.

Conforme informado em seção anterior deste trabalho, os parâmetros das séries temporais escolhidos RSCP e Ec/lo relacionam-se matemática da seguinte forma: $\operatorname{RSCP}(\mathrm{dBm})=$ RSSI $(\mathrm{dBm})+\mathrm{Ec} / \mathrm{lo}(\mathrm{dBm})$. A série temporal das amostras do Ec/lo se caracteriza pela estatítica descritiva apresentada na Tabela 13 e figuras 14 e 15.

Tabela 13 - Estatística descritiva para o parâmetro Ec/lo da nova série.

\begin{tabular}{c|c|c|c|c|c|c|c}
\hline VARIÁVEL & MÉDIA & DESVIO PADRÃO & VARIÂNCIA & VALOR MÍNIMO & VALOR MÁXIMO & ASSIMETRIA & CURTOSE \\
\hline Ec/lo & $-8,231$ & 1,817 & 3,302 & $-15,390$ & $-3,450$ & 0,43 & 1,07 \\
\hline
\end{tabular}

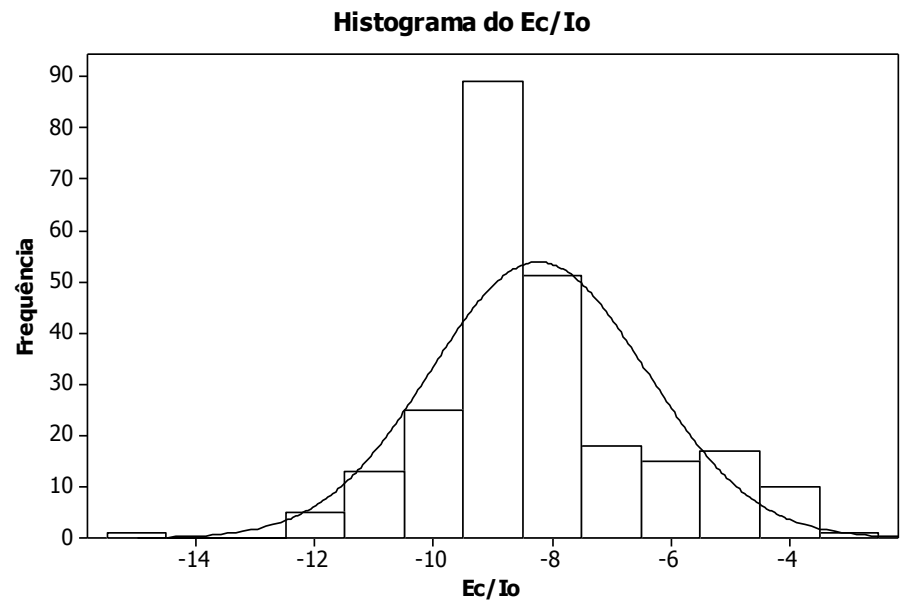

Figura 14 - Histograma do parâmetro Ec/lo. 


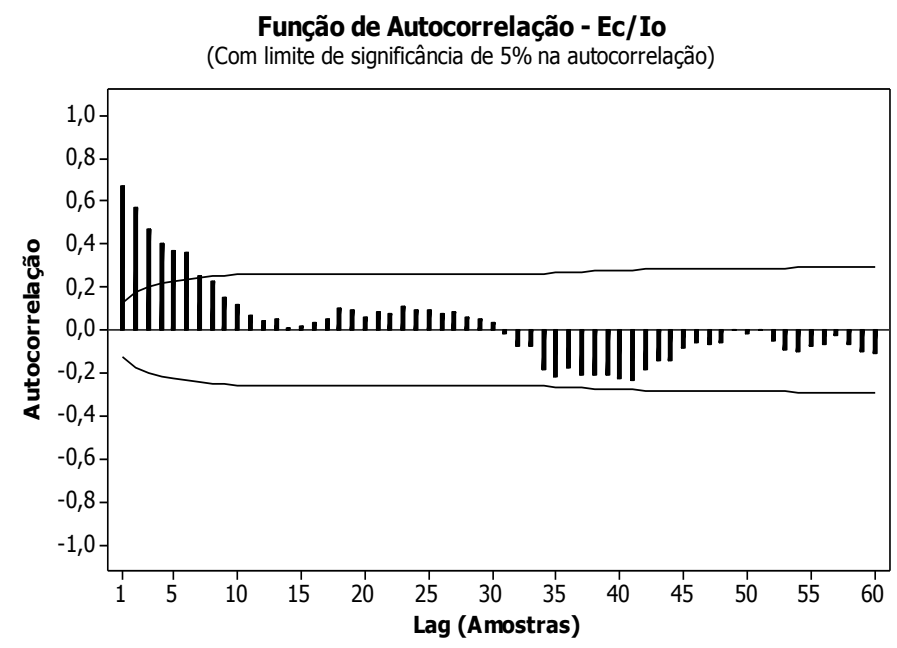

Figura 15 - Autocorrelação do parâmetro Ec/lo.

A predição de $10 \%$ (25) do volume de amostras (240) da nova série temporal Ec/lo é apresentada na Figura 16.

Alisamento Exponencial de Holt - Ec/Io

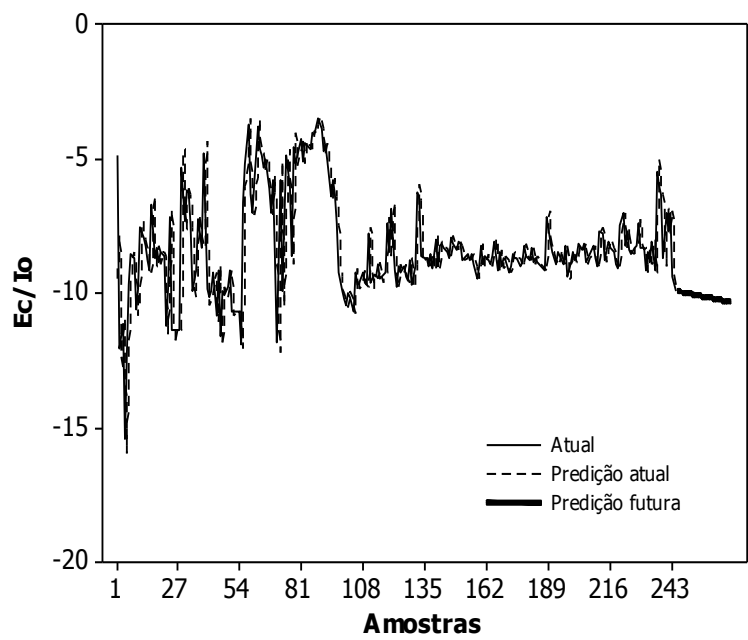

Figura 16 - Predição para a nova série de Ec/lo usando o Modelo de Holt.

A série temporal das amostras do RSCP se caracteriza pela estatítica descritiva apresentada na Tabela 14 e figuras 17 e 18.

Tabela 14 - Estatística descritiva para o parâmetro RSCP da nova série.

\begin{tabular}{c|c|c|c|c|c|c|c}
\hline VARIÁVEL & MÉDIA & DESVIO PADRÃO & VARIÂNCIA & VALOR MÍNIMO & VALOR MÁXIMO & ASSIMETRIA & CURTOSE \\
\hline RSCP & $-82,87$ & 7,59 & 57,67 & $-104,97$ & $-67,42$ & $-0,57$ & 0,44 \\
\hline
\end{tabular}




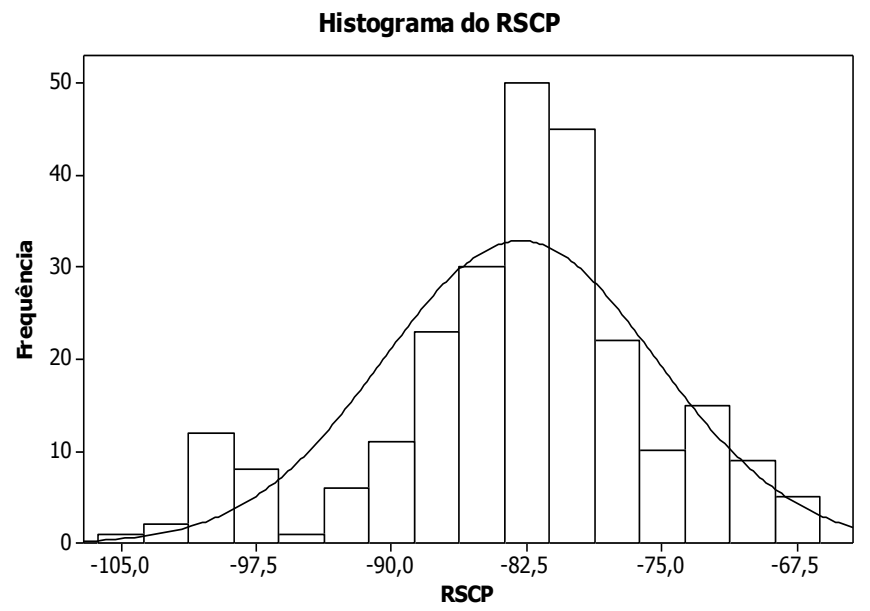

Figura 17 - Histograma do parâmetro RSCP.

Função de Autocorrelação - RSCP (Com limite de significância de 5\% na autocorrelação)

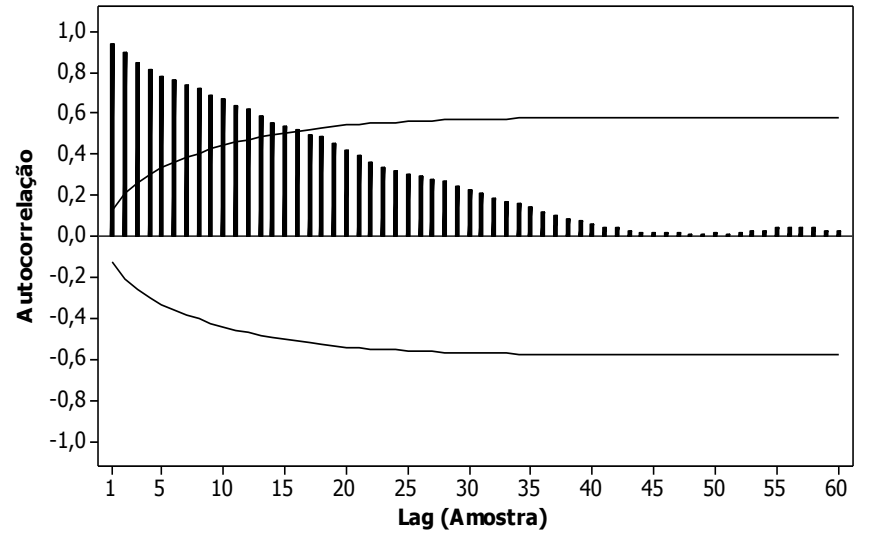

Figura 18 - Autocorrelação do parâmetro RSCP.

A predição de $10 \%$ (25) do volume de amostras (240) da nova série temporal Ec/lo é apresentada na Figura 19.

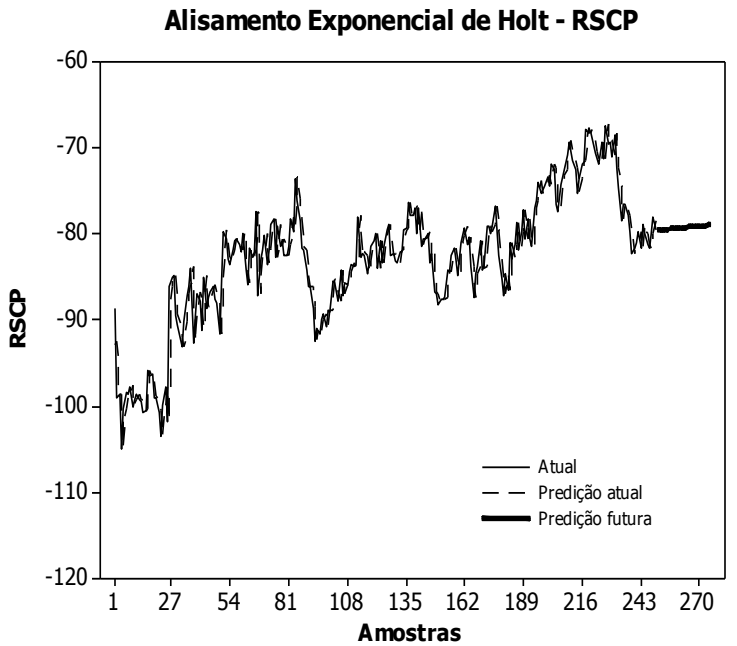

Figura 19 - Predição para a nova série de Ec/lo usando o Modelo de Holt. 


\section{CONCLUSÕES}

As séries de RSCP e Ec/lo têm correlação de Pearson no valor de 0,177 (fraca correlação), portanto, indica que as séries quase não têm dependência linear uma da outra, mas pode existir uma dependência não linear que aqui não será analisada por não ser o foco deste trabalho. Para realização do cálculo da correlação de Pearson foram desconsideradas cinco amostras (2\%) da série RSCP para ficar com a mesma quantidade de amostras (245) da série Ec/lo.

Armstrong \& Collopy (1992), também ressaltam apreferência de escolha das medidas MAD, MSD e MAPE em métodos de previsão.

Quanto menor o valor de MAPE melhor o ajuste do modelo. Para as duas séries externas RSCP (MAPE=2,44724) e EC/lo (MAPE=13,1111) os resultados de precisão dos métodos (MAPE) são coerentes com estudos empíricos prévios [22] que variam entre $3 \%$ e $13 \%$. Para os valores de MAD, em geral, compara-se o valor do erro acumulado com o valor de 4. Quando ultrapassar este valor, o problema deve ser identificado e o modelo deve ser revisto. Obtivemos modelos para a séries externas do RSCP com as medidas de acurácia (MAD=2,02680) e Ec/lo (MAD=0,9807).

A metodologia de suavização exponencial de Holt, um dos métodos de maior complexidade, foi abordado por ter a capacidade de modelar de forma satisfatória grande parte das séries temporais existentes em situações práticas (BIANCHI, 1993).

Diante das medidas de acurácia obtidas neste trabalho, podemos afirmar que o modelo de Alisamento Exponencial de Holt é indicado para análise, modelagem e previsão de boa parte de séries temporais de parâmetros (Rx Power, RSCP e Ec/lo) WCDMA/HSPA aqui apresentadas e em especial as séreis temporais RSCP e Ec/lo oriundas das amostras de no drive test.

O parâmetro Throughput tem as medidas de acurácia que divergem bastante das medidas de acurácia das séries temporais Rx Power, RSCP e Ec/lo, isso por ter características estatisticas bem distintas com altos valores para médias, variância e desvio padrão, também podemos observar que suas séries têm várias amostras com o valor zero durante alguns trajetos do drive test, se trata de um parâmetro que medi a velocidade de tráfego de dados que opera por rajadas de dados não deterministicas na rede WCDMA/HSPA.

A predição futura de parâmetros medidos através de drive test na rede WCDMA/HSPA em pleno funcionamento dar uma visibilidade antecipada de áreas críticas em regiões urbanas com coberturas problemáticas que apresentem tendências de degradação da qualidade do sinal em ritmo muita vezes acelerado. Acões antecipadas podem ser tomadas pelos responsáveis por Otimização e Planejamento de investimentos das redes WCDMA/HSPA, evitando perda de receita e reclamações de clientes usuários nas companhias que operam as redes móveis celulares.

\section{REFERÊNCIAS BIBLIOGRÁFICAS}

1. ARMSTRONG, J. S.; COLLOPY, F. Error Measures For Generalizing About Forecasting Methods: Empirical Comparisons. International Journal of Forecasting. v.8, p. 69-80, 1992.

2. BARROS, M., Processos Estocásticos, Papel Virtual, Rio de Janeiro 2004.

3. BIANCHI, L.; JARRETT, J. E.; HANUMARA, R. C. Forecasting incoming calls to telemarketing 
centers. The Journal of Business Forecasting Methods \& Systems. v. 12, n. 2; p. 3-12, 1993.

4. BLOGH, J. S. and HANZO, L., Third Generation Systems and Intelligent Wireless NetworkingSmart Antennas and Adaptive Modulation, John Willey \& Sons, Chichester, 2002.

5. BOX, G. E. P., JENKINS, G. M., Time Series Analysis Forecasting and Control. Holden Day, California, 1976.

6. BOX, G. E. P., JENKINS, G. M. and REINSEL, G. C., Time Series Analysis Forecasting and Control. Prentice Hall, New Jersey, 1994.

7. CHATFIELD, C., Time-series forecasting, Chapmam \& Hall/CRC, Boca Raton, 2000.

8. CUI, T., et. al., First Order Adaptive IIR Filter for CQI Prediction in HSDPA, 2010, IEEE Transactions on Education, 1-5, 18-21.

9. FADER, P. S.; HARDIE, B. G. S.; ZEITHAMMER, R. Forecasting new product trial in a controlled test market environment. Journal of Forecasting. v. 22, 2003, p. 391-410.

10. HYNDMAN, R. J.; KOEHLER, A. B. Another Look at Measures of Forecast Accuracy. International Journal of Forecasting. v.22, p. 679-688, 2006.

11. KAARANEN, Heikki et al., UMTS NETWORKS: Architecture, Mobility and Services, John Wiley \& Sons Ltd, Inc., West Sussex, 2005.

12. KLOCKAR, L., SIMONSSON, A., GUNNARSSON, F., \& Borg, A., 'Channel Characterization and HSDPA Bit Rate Prediction of a Dense City Network', 2009, IEEE Transactions on Education, 15, 26-29.

13. MAKRIDAKIS, S.; WHEELRIGHT, S. C.; HYNDMAN, R. J. Forecasting - Methods and Applications. New York: John Wiley, 1998.

14. MONTGOMERY, D. C., JERNNINGS, C., L and KULAHCI, M., Introduction to Time Series Analysis and Forecasting, John Wiley \& Sons, New Jersey, 2008.

15. MORETTIN, P. A, TOLOI C. M. C., Modelos para Previsão de Séries Temporais, Instituto de Matemática Pura e Aplicada, Rio de Janeiro, 1981.

16. NOKIA (2003), HSDPA Solution, Nokia Group, Finland. *http://www.itu.int/ITUD/tech/NGN/Manual/ManualAddReferences/A_3_2_3.pdf

17. SALLENT, O., et. al., A roadmap from UMTS optimization to LTE self-optimization, IEEE Transactions on Education, 49 (6), 172-182, 2011.

18. TOUHEED, H., QUDDUS, A.U. and TAFAZOLLI, R., Predictive CQI reporting for HSDPA, (2008). IEEE Transactions on Education 1-5, 15-18.

19. ZHENG, Y., et. al., A modified ARIMA model for CQI prediction in LTE-based mobile satellite communications, 2012, IEEE Transactions on Education, 822-826, 23-25.

20. WEI, W. W. S., Time Series Analysis - Univariate and Multivariate Methods, Pearson Education, Boston, 2006.

21. WRIGHT, G.; LAWRENCE, M.; COLLOPY, F. The Role of validity of Judgement in Forecasting. International Journal of Forecasting. v. 12, n. 1, p. 1-8, 1996. 